\title{
Left Ventricular Contained Rupture: True or Pseudoaneurysm?
}

\author{
Monish S. Raut ${ }^{1}$ Arun Maheshwari ${ }^{1} \quad$ Sumir Dubey ${ }^{2}$ Ganesh Shivnani ${ }^{2}$ Arun Mohanty ${ }^{3}$ \\ Aman Makhija ${ }^{3}$ \\ ${ }^{1}$ Department of Cardiac Anesthesiology, Sir Ganga Ram Hospital, \\ New Delhi, India \\ ${ }^{2}$ Department of Cardiac Surgery, Sir Ganga Ram Hospital, \\ New Delhi, India \\ ${ }^{3}$ Department of Cardiology, Sir Ganga Ram Hospital, New Delhi, India \\ Address for correspondence Dr. Monish S. Raut, MD, FNB, \\ Department of Cardiac Anesthesiology, Sir Ganga Ram Hospital, \\ New Delhi 110060, India (e-mail: drmonishraut@gmail.com). \\ J Card Crit Care TSS 2017;1:40-44.
}

\begin{abstract}
Acute myocardial infarction leading to left ventricular wall rupture is a very lethal complication with the risk of sudden cardiac death. Such contained rupture presents as aneurysmal dilatation of ventricular wall on echocardiography. A false aneurysm is bound by the pericardium without any myocardial tissue and true aneurysm is a bulging

Keywords

- left ventricular

- rupture

- pseudoaneurysm of scarred myocardium. Pseudoaneurysm with contained rupture sealed by pericardium needs early surgical intervention. Here, the authors present similar case mimicking as pseudoaneurysm on echocardiography, but it was found out to be true aneurysm as surgical finding.
\end{abstract}

\section{Introduction}

Left ventricular wall rupture after acute myocardial infarction (MI) is a fatal complication with the risk of sudden cardiac death. Such contained rupture presents as aneurysmal dilatation of ventricular wall on echocardiography. Pseudoaneurysm with contained rupture sealed by pericardium needs early surgical intervention. Here, the authors present similar case mimicking as pseudoaneurysm on echocardiography, but it was found out to be true aneurysm as surgical finding. The authors also discuss pathophysiology and management of such potentially dreadful cases.

\section{Case}

A 56-year-old man presented with retrosternal chest pain since 5 days. Electrocardiogram suggested anterolateral wall ST-T changes (-Fig. 1) and chest X-ray showed cardiomegaly (-Fig. 2). Echocardiography revealed a large aneurysm of the left ventricular lateral wall with mild mitral regurgitation (-Videos 1, 2). Aneurysmal wall was thinned out. Coronary angiography was suggestive of significant disease of the left circumflex and obtuse marginal artery (-Fig. 3). Considering impending rupture, the patient was immediately scheduled for aneurysmal repair. After smooth induction of anesthesia, right femoral artery and vein were cannulated to institute cardiopulmonary bypass. Midline sternotomy was performed and left ventricular vent was inserted through the right superior pulmonary vein to decompress the left ventricle. Full flow on cardiopulmonary bypass was established. The heart was arrested using antegrade cardioplegia after applying aortic cross clamp. As the aneurysm was incised and examined, it was noticed that a thin layer of myocardium was forming the aneurysmal sac. Dacron patch $(5 \times 5 \mathrm{~cm})$ covering the area of infarction was sutured to normal myocardium by taking shallow bites through the epicardium and superficial layers of myocardium. Aneurysmal sac was approximated using pledgeted sutures. After adequate de-airing, the patient could be weaned off cardiopulmonary bypass using adrenaline infusion support $(0.1 \mu \mathrm{g} / \mathrm{kg} / \mathrm{min})$. Postoperative transesophageal echocardiography showed left ventricular ejection fraction $40 \%$ with mild mitral regurgitation ( - Videos 3, 4; and - Fig. 4). Further course of the patient remained uneventful. The patient was discharged on seventh postoperative day in a stable condition.
DOI https://doi.org/ $10.1055 / \mathrm{s}-0037-1604329$.
Copyright (๑) 2017 Official Publication of License terms

The Simulation Society (TSS), accredited by International Society of

Cardiovascular Ultrasound (ISCU) 


\section{Video 1}

Transthoracic echocardiography showing large left ventricular lateral wall aneurysm. Online content including video sequences viewable at: www.thiemeconnect.com/ejournals/html/doi/10.1055/s-00371604329.

\section{Video 2}

Transesophageal echocardiography transgastric view showing large left ventricular lateral wall aneurysm. Online content including video sequences viewable at: www.thieme-connect.com/ejournals/html/doi/ 10.1055/s-0037-1604329.

\section{Video 3}

Transesophageal echocardiography transgastric view showing large left ventricular lateral wall defect closed by Dacron patch with obliteration of aneurysmal cavity. Online content including video sequences viewable at: www.thieme-connect.com/ejournals/ html/doi/10.1055/s-0037-1604329.

\section{Video 4}

Transesophageal echocardiography four-chamber view showing large left ventricular lateral wall defect closed by Dacron patch with obliteration of aneurysmal cavity. Online content including video sequences viewable at: www.thieme-connect.com/ ejournals/html/doi/10.1055/s-0037-1604329.

\section{Discussion}

Left ventricular free wall rupture is an uncommon but potentially fatal complication of MI. The incidence of left ventricular rupture after acute $\mathrm{MI}$ is more in the first 7 days. ${ }^{1}$ Free wall rupture of the infarcted ventricle is seen in 15 to $25 \%$ of the patients who died of $\mathrm{MI}^{2-4}$ However, it is observed in less than $1 \%$ of living patients with acute MI. ${ }^{5}$ Incomplete rupture constitutes 10 to $15 \%$ of total free wall ruptures. $^{6}$

Left ventricular free wall rupture after acute MI is 3 to 10 times more common than the rupture of the papillary muscle or ventricular septum. ${ }^{7}$ Most cases of such acute rupture are seen in the anterior and lateral wall, whereas subacute cardiac rupture is mainly observed with inferior MIs, as there is tendency of adherent blood clot formation in this region. ${ }^{8,9}$ Morphologically left ventricular rupture has been classified into four types. ${ }^{10}$ Type I constitutes little infiltration or dissection of the myocardium. Multicanalicular trajectory with extensive myocardial dissection is seen in type II. Type III left ventricular rupture is protected either by pericardial adhesion or by a thrombus sitting at the orifice on the ventricular side. Incomplete traversing of trajectory through all layers is observed in type IV.

An abrupt discontinuity in the ventricular wall with acute angle between the contour of the left ventricle and aneurysm is suggestive of pseudoaneurysm. ${ }^{11} \mathrm{~A}$ false aneurysm is bound by the pericardium without any myocardial tissue and more frequently seen in the inferolateral wall. ${ }^{12}$ True aneurysm is a bulging of scarred myocardium and more commonly observed in the anteroapical wall. ${ }^{12}$ Left ventricular aneurysms (LVAs) are commonly found in the anterior or apical walls in 70 to $85 \%$ of cases, most commonly due to total occluded left anterior descending coronary artery with absent collateralization.

Inferior-basal LVA walls due to right coronary artery occlusion are seen in 10 to $15 \%$ of the cases. However, lateral LVA, as in the present case, due to left circumflex artery blockage is exceedingly uncommon. ${ }^{13-15}$

Elderly, female patients with single severe coronary artery disease without any collateral and transmural MI are seen to be risk factors for developing left ventricular pseudoaneurysm with contained rupture. ${ }^{16}$

Most cardiac ruptures are located in the middle third of the ventricle within $1 \mathrm{~cm}$ of the papillary muscles insertion due to wall stress and arrangement of muscle fibers. ${ }^{16}$ The patient with smaller transmural infarct with good myocardial contractility are more prone to rupture than larger transmural infarct with low myocardial contractility. The reason could be patients with preserved ejection fraction generate higher intracavitary pressure during systole. ${ }^{17}$ Increased activity of matrix metalloproteinases after infarction would cause expansion in necrotic area with risk of cardiac rupture. $^{16}$ These findings may have potential therapeutic tool in preventing cardiac rupture after MI. ${ }^{16,18}$

Complete free wall rupture in this case could have been prevented due to myocardial hypertrophy. The lack of early reperfusion treatment made this patient susceptible to develop LVA. Dissection of the lateral myocardial wall in the infarcted area resulted in splitting of the lateral wall with more inner part of the wall hung into ventricular cavity and outer thin part became dilated (-Figs. 5, 6). Such lateral dilatation with very thin outer covering was difficult to be differentiated into a true LVA or pseudoaneurysm on echocardiography.

LVA can potentially lead to serious complications such as ventricular arrhythmias, heart failure, and thromboembolism. Ventricular contraction causes paradoxical dilatation of the aneurysmal segment. This results in decrease in the effective cardiac output and rise in LV end-diastolic pressure. ${ }^{19}$ An increase in wall stress and thereby increase in oxygen demand could be detrimental in presence of 


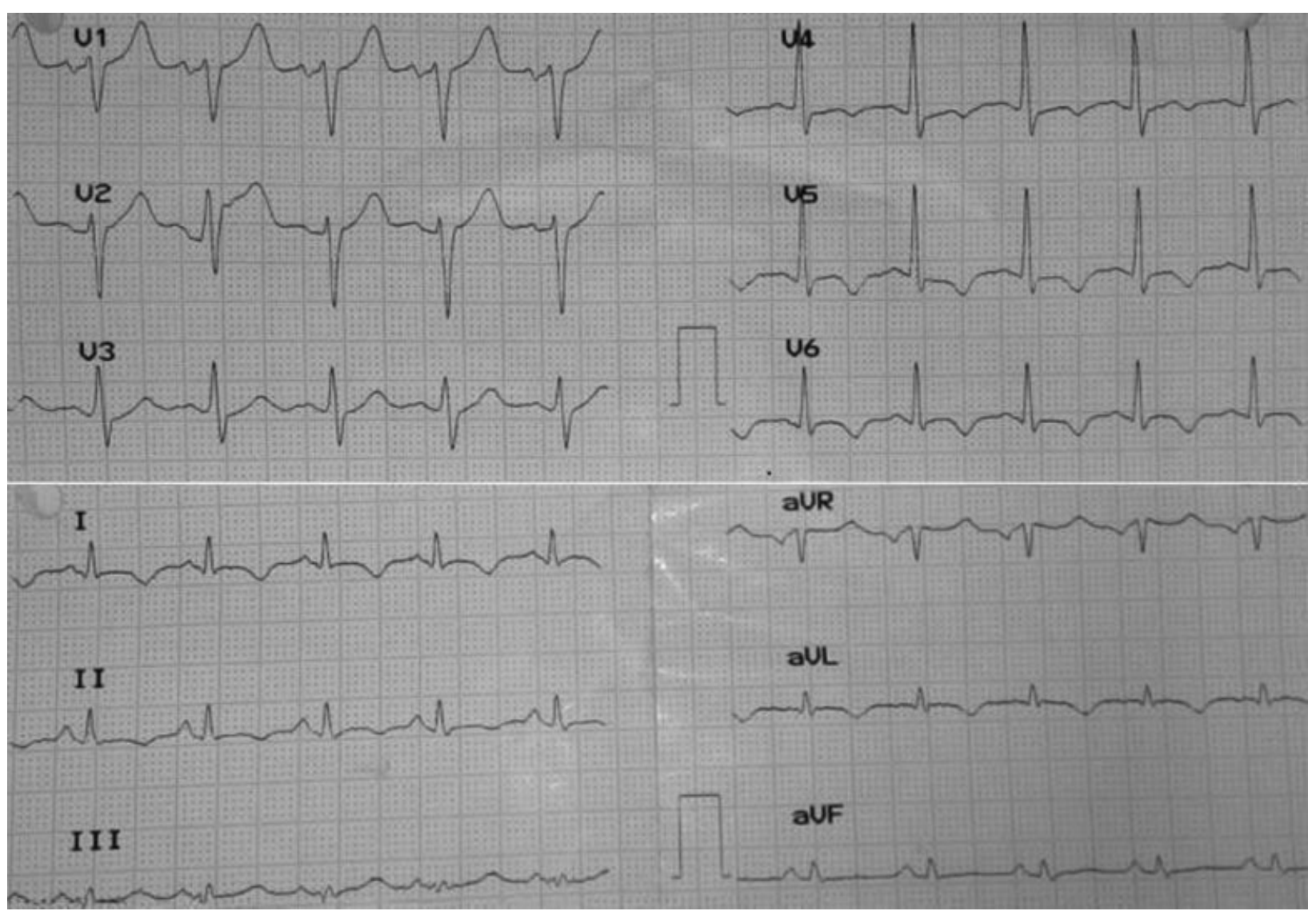

Fig. 1 ECG showing anterolateral T-wave inversions.

coronary artery disease and can cause further myocardial ischemia with angina. Long-standing left ventricular volume overload will result in left ventricular failure.

Myocardial stretch and fibrotic, damaged myocardial fibers can lead to enhanced triggered activity or reentrant tachycardia. ${ }^{20}$ In more than $50 \%$ of patients with LVA, a mural thrombus was found. ${ }^{21}$

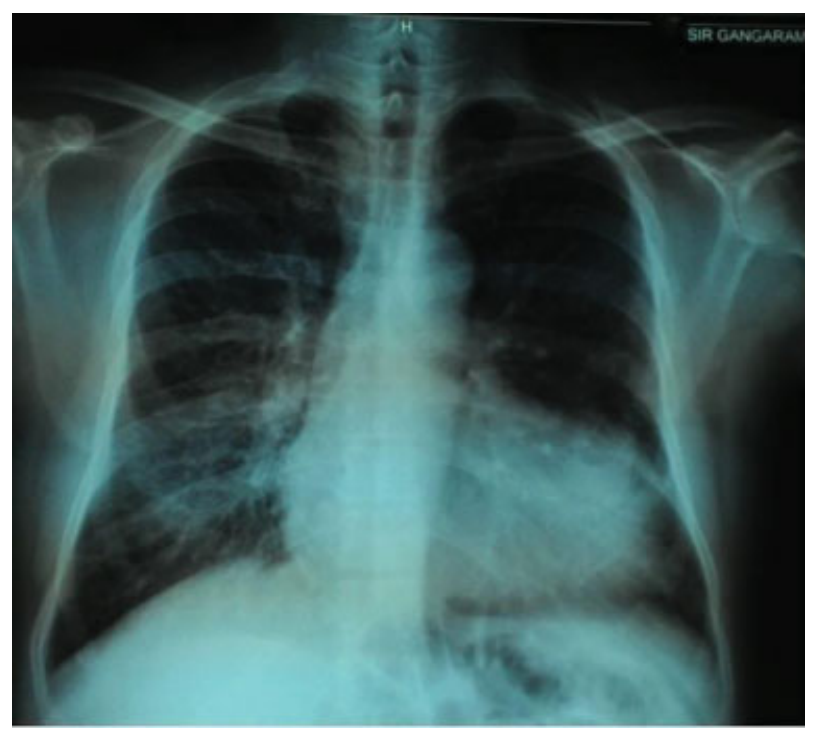

Fig. 2 Chest X-ray showing cardiomegaly.
Sensitivity of transthoracic echocardiography for diagnosis of subacute free wall rupture is approximately $70 \%{ }^{22}$ Cardiac magnetic resonance is a useful imaging modality to differentiate a true aneurysm from pseudoaneurysm with contained LV rupture after MI and to detect thrombus layered against the myocardium. ${ }^{23}$ Echocardiography of the present case revealed very thin aneurysmal covering presumed to be pericardium with contained rupture. Considering acute MI and impending rupture of pseudoaneurysm, the patient was urgently operated. However, surgical finding turned out to be true aneurysm as the sac wall was thinned myocardium.

Early improvement in the management and revascularization of patients with acute MI has decreased the incidence of LVA from $30-35 \%$ to $8-15 \%{ }^{24}$ The incidence of late rupture (after 4 days) might be reduced by reperfusion therapy. ${ }^{25}$

The patient with acute MI and at high risk of cardiac rupture should be managed crucially to prevent any such unfortunate event. Bedrest, strict control of systolic blood pressure, the use of sympatholytic $\beta$-blocking , and angiotensin-converting enzyme inhibitors (ACEIs) decrease wall stress and prevent the expansion of the necrotic area. ${ }^{16}$

Pseudoaneurysm has a greater tendency to rupture than true aneurysm. Untreated pseudoaneurysms carries the risk of rupture in 30 to $45 \%$ of the cases. ${ }^{26}$ Hence, most clinicians prefer surgical treatment for LV pseudoaneurysm. ${ }^{27}$ In a review of 290 patients with LV pseudoaneurysms by 

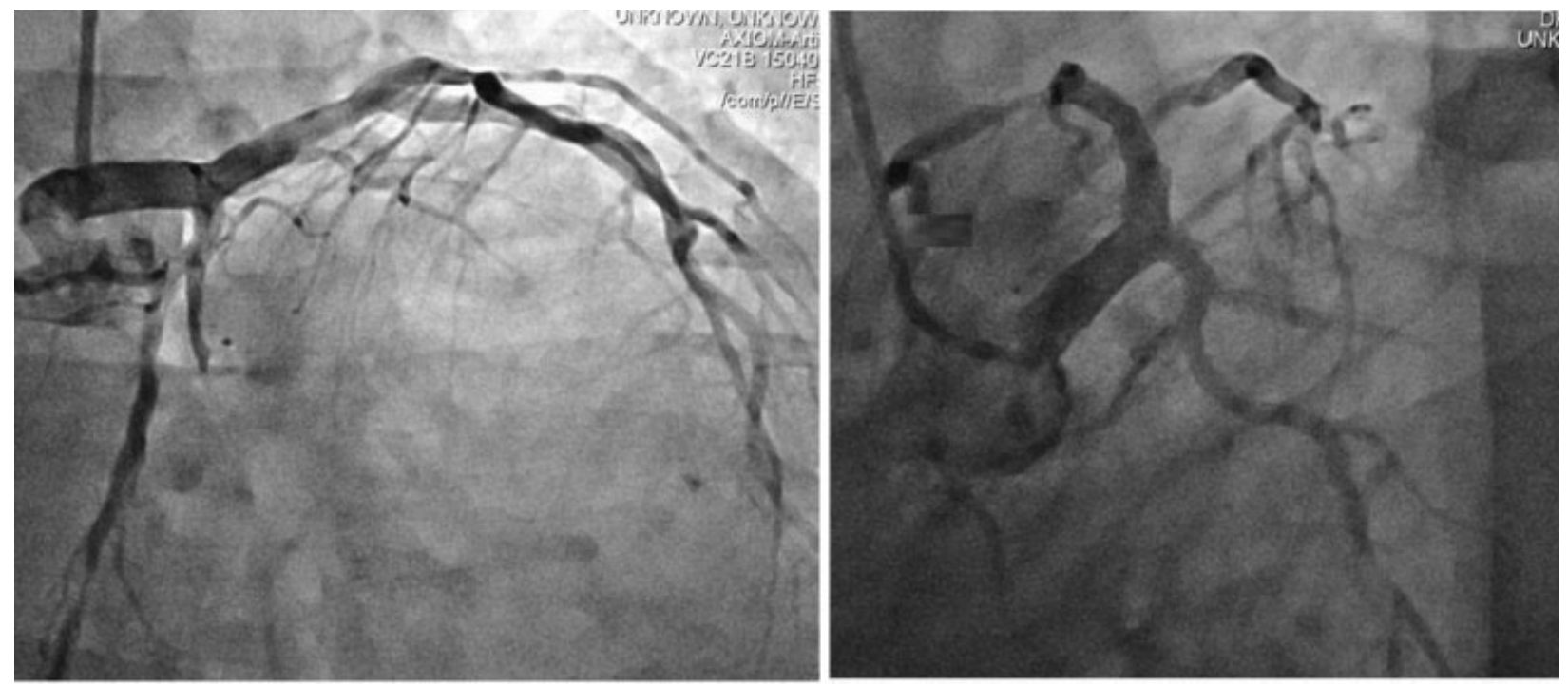

Fig. 3 Coronary angiography showing significant disease of left circumflex and obtuse marginal artery.

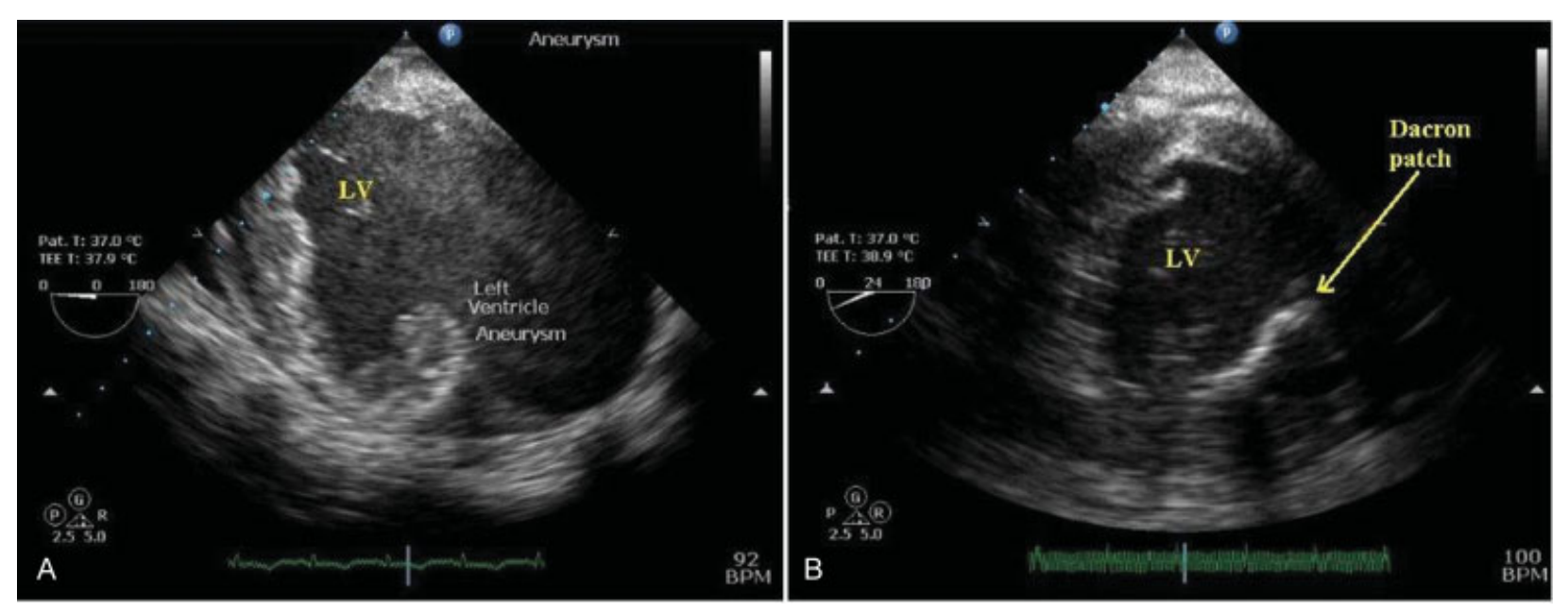

Fig. 4 Transesophageal echocardiography transgastric view before and after surgical repair. LV, left ventricle.

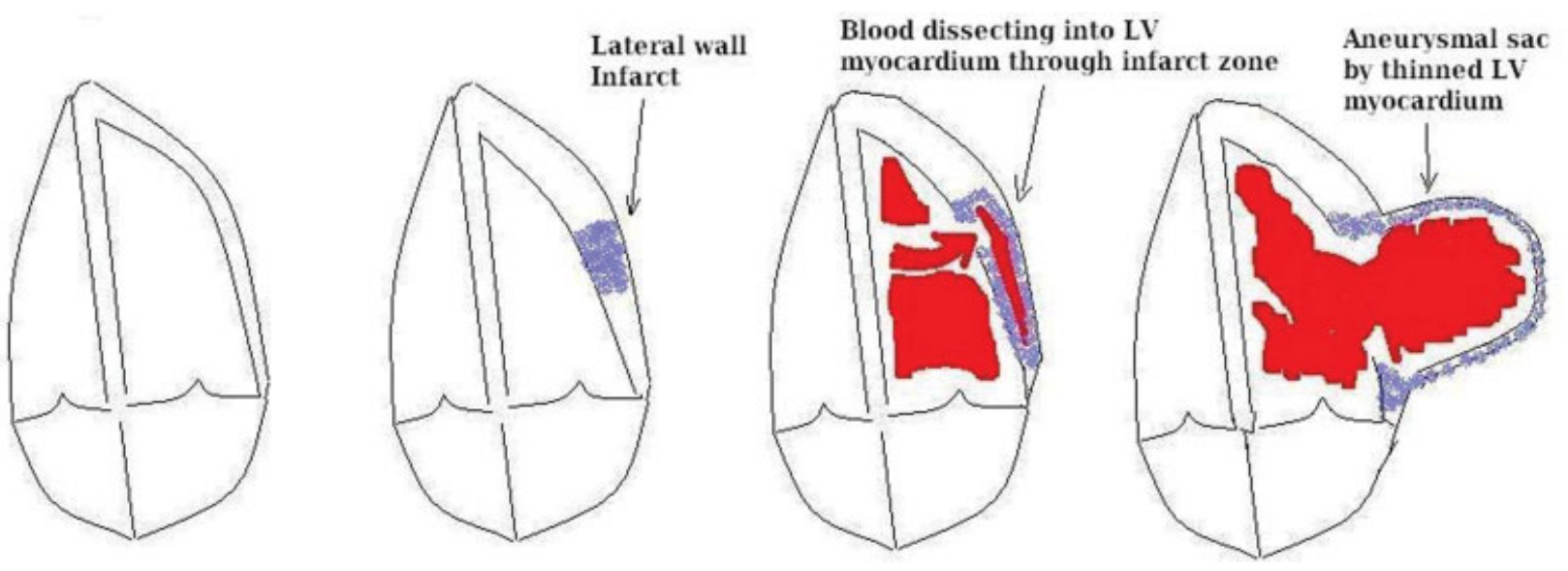

Fig. 5 Morphologic progression of left ventricular (LV) lateral wall aneurysm after infarction. 


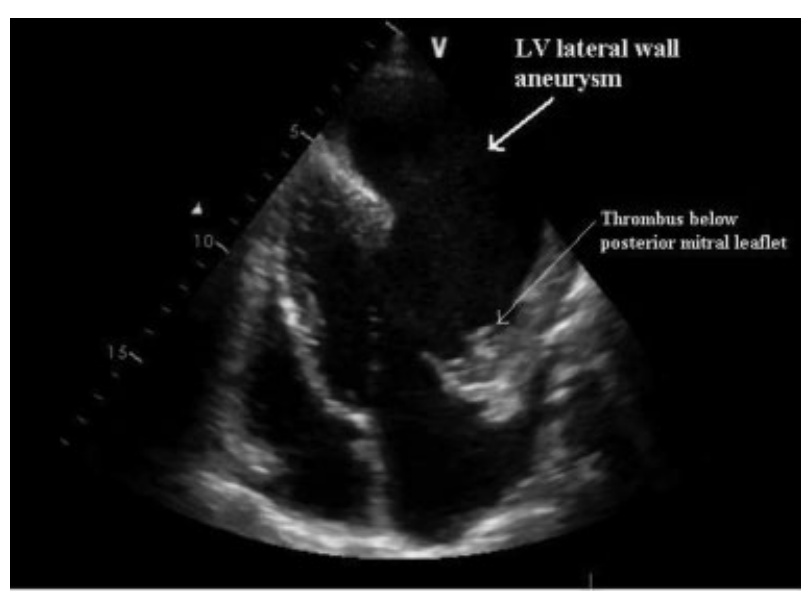

Fig. 6 Transthoracic echocardiography showing lateral wall aneurysm. LV, left ventricle.

Frances et al, ${ }^{27}$ surgically treated patients were having mortality rate of $23 \%$ and medically treated patients were having mortality rate of $48 \%$. Patients with organized thrombi within the pseudoaneurysm with a narrow neck can have good survival rate without surgery. ${ }^{11}$

Myocardial revascularization during the repair of cardiac rupture defect is not suggested as it does not improve the outcomes. ${ }^{16}$ It can be considered after 3 weeks after the acute episode. ${ }^{16}$ With current improved surgical techniques, perioperative mortality is reduced to less than $10 \%{ }^{27}$

\section{References}

1 Batts KP, Ackermann DM, Edwards WD. Postinfarction rupture of the left ventricular free wall: clinicopathologic correlates in 100 consecutive autopsy cases. Hum Pathol 1990;21(05):530-535

2 Reddy SG, Roberts WC. Frequency of rupture of the left ventricular free wall or ventricular septum among necropsy cases of fatal acute myocardial infarction since introduction of coronary care units. Am J Cardiol 1989;63(13):906-911

3 Stevenson WG, Linssen GC, Havenith MG, Brugada P, Wellens HJ. The spectrum of death after myocardial infarction: a necropsy study. Am Heart J 1989;118(06):1182-1188

4 Pohjola-Sintonen S, Muller JE, Stone PH, et al. Ventricular septal and free wall rupture complicating acute myocardial infarction: experience in the Multicenter Investigation of Limitation of Infarct Size. Am Heart J 1989;117(04):809-818

5 Becker RC, Gore JM, Lambrew C, et al. A composite view of cardiac rupture in the United States National Registry of Myocardial Infarction. J Am Coll Cardiol 1996;27(06):1321-1326

6 Slater J, Brown RJ, Antonelli TA, et al. Cardiogenic shock due to cardiac free-wall rupture or tamponade after acute myocardial infarction: a report from the SHOCK Trial Registry. Should we emergently revascularize occluded coronaries for cardiogenic shock? J Am Coll Cardiol 2000;36(3, Suppl A):1117-1122

7 Murphy JG. Mayo Clinic Cardiology Review. Armonk, NY: Futura Publishing; 1997:148

8 Purcaro A, Costantini C, Ciampani N, et al. Diagnostic criteria and management of subacute ventricular free wall rupture compli- cating acute myocardial infarction. Am J Cardiol 1997;80(04): 397-405

9 Exadaktylos NI, Kranidis AI, Argyriou MO. Left ventricular free wall rupture during acute myocardial infarction. Hellenic J Cardiol 2002;43:246-252

10 Perdigao C, Andrade A, Ribeiro C. [Cardiac rupture in acute myocardial infarction. Various clinico-anatomical types in 42 recent cases observed over a period of 30 months] [in French]. Arch Mal Coeur Vaiss 1987;80(03):336-344

11 Mao C-T, Li M-F, Kao Y-C, Cherng W-J, Hung M-J. Long-term survival of a patient with asymptomatic left ventricular pseudoaneurysm after acute myocardial infarction. J Intern Med Taiwan 2012;23:442-448

12 Higgins CB, Lipton MJ, Johnson AD, Peterson KL, Vieweg WV. False aneurysms of the left ventricle. Identification of distinctive clinical, radiographic, and angiographic features. Radiology 1978;127(01):21-27

13 Nagle RE, Williams DO. Proceedings: natural history of ventricular aneurysm without surgical treatment. Br Heart J 1974;36(10): 1037

14 Dubnow MH, Burchell HB, Titus JL. Postinfarction ventricular aneurysm. A clinicomorphologic and electrocardiographic study of 80 cases. Am Heart J 1965;70(06):753-760

15 Gorlin R, Klein MD, Sullivan JM. Prospective correlative study of ventricular aneurysm. Mechanistic concept and clinical recognition. Am J Med 1967;42(04):512-531

16 Soli SC, Pujol D, Mauro V. Left ventricular free wall rupture after acute myocardial infarction. Rev Argent Cardiol 2009;77:395-404

17 Tao ZY, Cavasin MA, Yang F, Liu YH, Yang XP. Temporal changes in matrix metalloproteinase expression and inflammatory response associated with cardiac rupture after myocardial infarction in mice. Life Sci 2004;74(12):1561-1572

18 Creemers EE, Cleutjens JP, Smits JF, Daemen MJ. Matrix metalloproteinase inhibition after myocardial infarction: a new approach to prevent heart failure? Circ Res 2001;89(03):201-210

19 Klein MD, Herman MV, Gorlin R. A hemodynamic study of left ventricular aneurysm. Circulation 1967;35(04):614-630

20 Waldo AL, Arciniegas JG, Klein H. Surgical treatment of lifethreatening ventricular arrhythmias: the role of intraoperative mapping and consideration of the presently available surgical techniques. Prog Cardiovasc Dis 1981;23(04):247-264

21 Reeder GS, Lengyel M, Tajik AJ, Seward JB, Smith HC, Danielson GK. Mural thrombus in left ventricular aneurysm: incidence, role of angiography, and relation between anticoagulation and embolization. Mayo Clin Proc 1981;56(02):77-81

22 López-Sendón J, González A, López de Sá E, et al. Diagnosis of subacute ventricular wall rupture after acute myocardial infarction: sensitivity and specificity of clinical, hemodynamic and echocardiographic criteria. J Am Coll Cardiol 1992;19(06):1145-1153

23 Karamitsos TD, Ferreira V, Banerjee R, Moore NR, Forfar C, Neubauer S. Contained left ventricular rupture after acute myocardial infarction revealed by cardiovascular magnetic resonance imaging. Circulation 2012;125(18):2278-2280

24 Glower DG, Lowe EL. Left ventricular aneurysm. In: Edmunds LH, ed. Cardiac Surgery in the Adult. New York, NY: McGraw-Hill; 1997:677

25 Mishra PK, Pathi V, Murday A. Post myocardial infarction left ventricular free wall rupture. Interact Cardiovasc Thorac Surg 2007;6(01):39-42

26 Vlodaver Z, Coe JI, Edwards JE. True and false left ventricular aneurysms. Propensity for the altter to rupture. Circulation 1975; 51(03):567-572

27 Frances C, Romero A, Grady D. Left ventricular pseudoaneurysm. J Am Coll Cardiol 1998;32(03):557-561 